\title{
In Search of the Optimal Oxygen Saturation for Extremely Low Birth Weight Infants: A Systematic Review and Meta-Analysis
}

\author{
Ola Didrik Saugstad ${ }^{a}$ Dagfinn Aune ${ }^{b}$

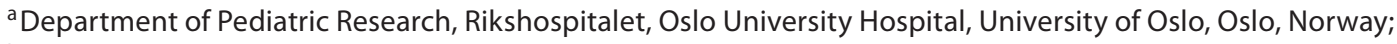 \\ ${ }^{b}$ Department of Epidemiology and Public Health, Imperial College, London, UK
}

\section{Key Words}

Arterial oxygen saturation • Bronchopulmonary dysplasia • Mortality $\cdot$ Pulse oximetry $\cdot$ Retinopathy of prematurity

\begin{abstract}
Background: The optimal arterial oxygen saturation in the first weeks of life is unknown for immature newborn infants. Objectives: To determine the effect of targeting high versus low oxygen saturation in the first weeks of life on the outcome of very low and extremely low birth weight infants. Methods: Randomized and observational studies were sought that compared the outcomes in babies with high or low oxygen saturation targeting assessed by pulse oximetry. Results: Ten studies were identified, of which 8 had severe retinopathy of prematurity $(n=3,811)$ and 8 had bronchopulmonary dysplasia/lung problems ( $n=4,612)$ as outcomes. Two studies also provided survival data. The relative risk (RR) in favor of low $\mathrm{SpO}_{2}$ was $0.42(95 \% \mathrm{Cl} 0.34-0.51)$ for severe retinopathy of prematurity, $0.73(95 \% \mathrm{Cl} 0.63-0.86)$ for bronchopulmonary dysplasia/lung problems, and $1.12(95 \% \mathrm{Cl}$ 0.86-1.45) for mortality. There was 1 randomized trial with retinopathy of prematurity, 3 with bronchopulmonary dysplasia/lung problems, and 1 with mortality as the outcome. When analyzing the randomized trial separately, the RR $(95 \%$ $\mathrm{Cl})$ for severe retinopathy of prematurity was $0.48(0.34-$
\end{abstract}

0.68), for bronchopulmonary dyslasia/lung problems it was 0.79 (0.64-0.97), and for mortality it was 1.27(1.01-1.60). Conclusions: A low oxygen saturation approach reduces severe retinopathy of prematurity by $50 \%$, i.e. from 20.9 to $9.5 \%$, and bronchopulmonary dysplasia/lung problems by $25 \%$, i.e. from 40.8 to $29.7 \%$. Further randomized trials are needed to provide definite conclusions and to assess whether reducing oxygen saturation has an impact on mortality among very and extremely low birth weight infants.

Copyright $\odot 2010$ S. Karger AG, Basel

\section{Introduction}

The optimal oxygen saturation in the first postnatal weeks for very low and extremely low birth weight infants has not yet been defined. This is in spite of the fact that hyperoxia has been postulated for several decades to be an important pathogenetic factor both for retinopathy of prematurity (ROP) and bronchopulmonary dysplasia (BPD) or chronic lung disease. When the association between high oxygen supplementation and retrolental fibroplasia (later called ROP) was detected in the 1950s, it was thought that this condition was understood and could be prevented [1]. However, in the 1970s and 1980s it was clear that ROP has not been eradicated; by contrast,

\section{KARGER}

Fax +41613061234 E-Mail karger@karger.ch www.karger.com (c) 2010 S. Karger AG, Basel

$1661-7800 / 11 / 1001-0001 \$ 38.00 / 0$

Accessible online at:

www.karger.com/neo
Prof. Ola Didrik Saugstad

Pediatrisk Forskningsinstitutt

Oslo Universitets Sykehus, Rikshospitalet, PB 4950 Nydalen

$\mathrm{NO}-0424$ Oslo (Norway)

Tel. +47 2307 2790, Fax +47 2307 2780, E-Mail odsaugstad@ rr-research.no 
the rate was increasing mainly due to the survival of more immature babies [2]. The BPD rate also persisted at a high level in spite of treatment with antenatal steroids, postnatal surfactant, and closer oxygen control [3].

A big leap forward in the understanding of the pathogenesis of these conditions occurred when we, in the 1980s, began to understand the concept of oxidative stress in preterm infants. A major breakthrough was in fact achieved when it was understood that oxidative stress is not related to oxygen treatment only [4]. For instance, the understanding that inflammation and oxidative stress are 2 sides of the same coin was extremely useful for understanding oxidative stress-related conditions, especially BPD $[5,6]$. At that time it therefore became clear that premature infants are more exposed to high oxidative stress not only because they are exposed to oxygen therapy but also because they: (1) are exposed to inflammation, (2) have a poorly developed defense against oxidative stress, and (3) often have free iron in tissues which triggers the production of hydroxyl radicals [4]. This knowledge contributed to new attention and a new approach to preventing oxygen radical-related conditions in the newborn or the so-called oxygen radical disease of the newborn [7]. However, since therapies with different antioxidants so far have not been very useful, the most rational approach to reducing oxidative stress is to control oxygen supplementation and prevent inflammation [8]. Other potential oxidative stress-promoting factors such as blood transfusions and the infusion of parenteral nutrition should also be taken into consideration $[9,10]$.

The development of pulse oximetry made it possible to systematically monitor arterial oxygen saturation $\left(\mathrm{SpO}_{2}\right)$ continuously [11]. A new interest in $\mathrm{SpO}_{2}$ levels and their control was aroused when Tin et al. [12] published in 2001 their observational study from Northern England showing that targeting a high $\mathrm{SpO}_{2}$ is associated with increased morbidity such as ROP and lung problems. Since then, a number of articles have been published showing that the rates of ROP and $\mathrm{BPD}$ are reduced when $\mathrm{SpO}_{2}$ is low compared to when it is high [for review see 13, 14]. However, a recent study by Chen et al. [15] indicates that this is valid for ROPduring the first weeks of life only. After approximately 32 weeks of postconceptional age $\mathrm{SpO}_{2}$ should perhaps be kept higher in order to prevent the progress of ROP. The recently published SUPPORT trial [16] found a significant reduction in ROP in low compared with high saturation targeted babies. This trial raised, however, concern about low $\mathrm{SpO}_{2}$ targeting because a small, but significant, increase in mortality was found in the low $\mathrm{SpO}_{2}$ target group.
In the present article a systematic review and metaanalysis of the studies published regarding high or low $\mathrm{SaO}_{2}$ targeting and outcomes such as ROP, BPD, and mortality is presented.

\section{Methods}

By searching PubMed and Embase up to July 2010, using the search words 'oxygen saturation and bronchopulmonary dysplasia' and 'oxygen saturation and retinopathy of prematurity', searching the Cochrane library and the literature, and looking for abstracts from major meetings, we have been able to identify 14 references including 15 studies examining the relation between high or low $\mathrm{SpO}_{2}$ and ROP and/or BPD. Five studies were excluded as described below.

Wallace at al. [17] presented the results in terms of the number of eyes only, not in terms of individuals like the other studies did; therefore, this study was excluded. However the study found that low versus high $\mathrm{SpO}_{2}$ groups had a nonsignificant reduction from 18 to $14 \%$ in severe ROP.

Britt and Sandoval published an abstract referred to by Wallace et al. [17] on babies $\leq 30$ weeks old with low (85-93\%) versus high (not specified) $\mathrm{SpO}_{2}$ showing a reduction in ROP stage 3 or more from $18 / 37$ (49\%) to $5 / 33$ (15\%). This study is not included due to lack of basic information.

Anderson et al. [18] conducted a survey showing that severe ROP was diagnosed in $5.7 \%$ of units with $\mathrm{SpO}_{2}$ targeting $>92 \%$ and in $2.5 \%$ of those with $\mathrm{SpO}_{2}<93 \%$. The study did not give exact numbers; it only presented the percentage of severe ROP and was therefore excluded.

Wright et al. [19] collected data prospectively from 3 neonatal intensive care units in the Los Angeles region. A protocol aimed at keeping the $\mathrm{SpO}_{2}$ levels between 83 and $93 \%$ and avoiding fluctuations was implemented between 1998 and 2002. Data were collected in babies with a birth weight between 500 and $1,500 \mathrm{~g}$. $\mathrm{SpO}_{2}$ policies before 1998 varied between hospitals with levels of 89-94, $90-95$, and $>90 \%$ in the 3 hospitals, respectively. The threshold ROP decreased from 7.3 to $1.3 \%$ after the change. However, there is an overlap of patients in this study and the study by the Noori et al. [20]. Which patients who are overlapping cannot be determined from the articles, and the data from Wright et al. [19] were therefore excluded.

Sears et al. [21] conducted a study that included a patient cohort which from 2005 to 2006 received the standard oxygen supplementation protocol. This cohort had oxygen targets of 95$100 \%$ saturation compared with a patient cohort from 2006 to 2007 which had strictly monitored oxygen targets of $<34$ weeks corrected gestational age oxygen limits of $80-95 \%$ with a target of $85-92 \%$ oxygen saturation, and $>34$ weeks corrected gestational age limits of $85-100 \%$ with a target of $92-97 \%$ saturation. ROP stage 3 decreased from 11 to $2 \%$. The study is not included because $\mathrm{SpO}_{2}$ targets were increased when the postconceptional age exceeded 34 weeks.

Below is a brief description of the 10 remaining studies, with basic information summarized in table 1 .

STOP ROP was a randomized study with the objective of determining the efficacy and safety of supplemental therapeutic ox- 
Table 1. Characterization of the studies

\begin{tabular}{lllcc}
\hline $\begin{array}{l}\text { Study } \\
\text { (first author, year) }\end{array}$ & $\begin{array}{l}\text { Gestational age or } \\
\text { birth weight }\end{array}$ & Study design & $\mathrm{High} \mathrm{SaO}_{2}$ & $\mathrm{Low} \mathrm{SaO}_{2}$ \\
\hline $2000[22]$ & & Randomized & $96-99$ & $89-94$ \\
Tin, 2001 [12] & $<28$ weeks & Observational & $88-98$ & $70-90$ \\
Sun, 2002 [24] & $500-1,000 \mathrm{~g}$ & Survey & $>95$ & $\leq 95$ \\
Askie, 2003 [23] & $<30$ weeks & Randomized & $95-98$ & $91-94$ \\
Chow, 2003 [27] & $500-1,500 \mathrm{~g}$ & Observational & $90-98$ & $85-93$ \\
Vanderveen, 2006 [26] & $\leq 28$ weeks or $\leq 1,250 \mathrm{~g}$ & Historical control & $87-97$ & $85-93$ \\
Deulofeut, 2006 [25] & $\leq 1,250 \mathrm{~g}$ & Historical control & $92-100$ & $85-93$ \\
Noori, 2009 [20] & $<1,000 \mathrm{~g}$ & Historical control & $89-94$ & $83-89$ \\
Tokuhiro, 2009 [28] & $<33$ weeks & Historical controls & $92-98$ & $88-92$ \\
SUPPORT, 2010 [16] & $24-28$ weeks & Randomized & $91-95$ & $85-89$ \\
\hline
\end{tabular}

ygen for infants with prethreshold ROP [22]. The hypothesis was that high oxygen supplementation reduces progression from prethreshold ROP. Six hundred forty-nine patients with confirmed prethreshold ROP in at least 1 eye were enrolled between 1994 and 1999 from 30 centers in the USA and randomized to a conventional (low) arm with $\mathrm{SpO}_{2}$ targeted at $89-94 \%$, or a supplementary arm (high) with pulse oximetry targeted at 96-99\%, for at least 2 weeks. The mean gestational age was 25.4 weeks and enrollment occurred, on average, 10 weeks later. Three hundred twentyfour patients were enrolled in the supplementary group and 325 in the conventional oxygen saturation group; of these patients, 30 and 36 were excluded from calculations of pulmonary complications defined as pneumonia and/or exacerbations of chronic lung disease which occurred in $8.5 \%$ of cases in the low saturation group versus $13.2 \%$ of cases in the high saturation group $(\mathrm{p}<$ 0.06 ). Data for ROP are not included because ROP was an inclusion criterion.

Tin et al. [12] examined the case notes of all babies born between 1990 and 1994 who survived infancy after delivery before 28 weeks of gestation in Northern England. They were able to identify 4 strategies for targeting $\mathrm{SpO}_{2}$, i.e. a high, a low, and 2 intermediate strategies. The high strategy kept $\mathrm{SpO}_{2}$ alarms between 88 and $98 \%$ for at least the first 8 weeks of life and the low $\mathrm{SpO}_{2}$ strategy kept $\mathrm{SpO}_{2}$ alarm limits between 70 and $90 \%$. The staff aimed to maintain saturation in the top half of the target range. Sixty-five infants survived in each group with a mean gestational age between 26 and 27 weeks. In the high $\mathrm{SpO}_{2}$ group $27.7 \%$ developed severe ( $\geq$ stage $3+$ ) ROP versus $6.2 \%$ in the low group. In the high $\mathrm{SpO}_{2}$ group $46 \%$ were on oxygen at 36 weeks postmenstrual age versus $18 \%$ in the low $\mathrm{SpO}_{2}$ group. The 1-year survival was $65 / 123(52.8 \%)$ in the high group versus $65 / 126$ $(51.6 \%)$ in the low group. This difference was not significant.

The BOOST study by Askie et al. [23] was a double-blinded randomized multicenter study from Australia enrolling 358 infants from 1996 to 2000 born at less than 30 weeks of gestation who remained dependent on supplemental oxygen at 32 weeks of postmenstrual age. Infants were randomly assigned to a target standard $\mathrm{SpO}_{2}$ of $91-94 \%(\mathrm{n}=178)$ or a high saturation group with $\mathrm{SpO}_{2}$ from 95 to $98 \%(\mathrm{n}=180)$; this target was maintained for the duration of the supplemental-oxygen therapy. The high saturation group received oxygen for a longer period after randomiza- tion (40 vs. 18 days, $\mathrm{p}<0.001)$ and had a significantly higher rate of BPD defined as dependence on supplemental oxygen at 36 weeks of postmenstrual age ( 64.4 vs. $46.1 \%, p<0.001)$. Because of the late randomization, the ROP data from this study are not included.

Sun et al. [24] performed a national survey among hospitals participating in the Vermont Oxford Network in the USA. Premature infants with a birth weight between 500 and $1,000 \mathrm{~g}$ born from 1998 to 2000 were included. The babies were divided into 2 groups: those who were exposed to a target $\mathrm{SpO}_{2}$ upper limit $>95 \%$ and those with an $\mathrm{SpO}_{2} \leq 95 \%$. In total 1,544 infants from 7 centers were included; 742 infants were in the high arm and 802 in the low arm. Severe ROP (stage $\geq 3$ ) was found in $29 \%$ of babies in the high group and in $10 \%$ of babies in the low group. Chronic lung disease, defined as $\mathrm{O}_{2}$ requirement at or beyond 36 weeks of postconceptional age, was twice as high in the high $\mathrm{SpO}_{2}$ group (51.2\%) versus the low $\mathrm{SpO}_{2}$ group (27.3\%) $\mathrm{p}<0.001$.

Deulofeut et al. [25] compared the rates of BPD and other oxygen-related conditions in babies with a weight $\leq 1,250$ g enrolled in a single center before and after they changed from a high saturation regime with $\mathrm{SpO}_{2}$ alarms between 92 and $100 \%$ to a regime to avoid hyperoxia with a target $\mathrm{SpO}_{2}$ of $85-93 \%$. They compared 300 infants born between 2000 and 2002 with 202 infants born from 2003 to 2004 . The mean gestational age was about 27 weeks. Severe ROP (stages 3 and 4 ) was found in $7.2 \%$ of cases in the high saturation period versus $4.6 \%$ in the low saturation period. In the first period with a high oxygen approach $51 \%$ of patients developed chronic lung disease defined as oxygen dependency at 36 weeks of postconceptional age versus $35 \%$ of patients in the low saturation group in the last period.

This study also showed a significantly higher mental developmental index in the last versus the first period $89.2 \pm 18.5$ vs. 80.2 \pm 18.3 .

Vanderveen et al. [26] examined their results regarding ROP before and after changing their oxygen saturation alarm limits. Before June 2003, alarm limits were set at $87-97 \%$ without a specified target range. After this date the alarm limits were lowered to 85-93\% with the target set at $90-92 \%$ for infants with a birth weight $\leq 1,250$ g or aged 28 weeks or less. Prethreshold ROP occurred in $17.5 \%$ of infants 3 years before the change (the high $\mathrm{SpO}_{2}$ 
period) compared with $5.6 \%$ the year after the change (the low saturation period).

Chow et al. [27] collected data prospectively during a 5-year period in a single Los Angeles tertiary center from 1997 to 2001 in infants with a birth weight between 500 and 1,500 g. Before $1998, \mathrm{SpO}_{2}$ limits were typically $90-98 \%$. In 1998 a new policy for $\mathrm{O}_{2}$ monitoring and administration during the first 4-8 weeks of life was implemented. The main objectives of this protocol were to avoid hyperoxia and repeated episodes of hypoxia-hyperoxia. In babies $\leq 32$ weeks old the accepted $\mathrm{SpO}_{2}$ were from 85 to $93 \%$, and the accepted $\mathrm{SpO}_{2}$ were $85-95 \%$ in babies $>32$ weeks old. There was a decrease in the incidence of severe ROP (stages $\geq 3$ ) from 1997 to 2001 , i.e. from 12.5 to $2.5 \%$. The rates of severe ROP were calculated using the number of diagnosed cases of severe ROP as the numerator and the total number of infants who received retinal examinations as the denominator. These numbers are not presented in the article so an estimated rate is given by the number of severe ROP divided by the number of infants surviving until discharge. In 1997 and 2001 the number of children surviving until discharge with a body weight of 500-1,500 g was 75 and 77 , respectively. The rate of severe ROP was estimated to be $9 / 75$ in 1997 and 2/77 in 2001.

Noori et al. [20] studied ROP and BPD in extremely low birth weight infants in 2 hospitals in the Los Angeles region before and after a reduction of $\mathrm{SpO}_{2}$ in 2002. Before the change the infants were managed with an $\mathrm{SpO}_{2}$ target of $89-94 \%$. After the change the $\mathrm{SpO}_{2}$ limits were $83-89 \%$. After 33 postmenstrual weeks the target $\mathrm{SpO}_{2}$ were between 90 and 94\%. Infants born between 1998 and 2001 were compared with infants born from 2003 to 2006, excluding babies born in 2002 . ROP $\geq$ stage 3 decreased, however, from 50.7 to $15.7 \%$. BPD at 36 weeks postmenstrual age was found in $44.9 \%$ of cases in the high saturation period versus $46.6 \%$ in the low saturation period. After the implementation of the new guidelines there was an increase in the incidence of hemodynamically significant ductus arteriosus [OR 1.77, 95\% confidence interval (CI) 1.03-3.06]; however, there was no significant difference in the rate of surgical ligation.

Tokuhiro et al. [28] performed at retrospective analyses in babies $<33$ weeks of gestational age from a single hospital in Japan. Between July 1, 2004, and December 31, 2005, the $\mathrm{SpO}_{2}$ alarm limits were set at 92 and 98\%. From January 1, 2006, on the $\mathrm{SpO}_{2}$ alarm limits were 88 and $92 \%$ and babies $<33$ weeks of gestational age were included until October 31, 2007. Fifty-nine and 78 children were included in the 2 periods, respectively. ROP stages 3 and 4 decreased from 32.2 to $17.9 \%$ from the first to the second period. Chronic lung disease decreased from 16.9 to $10.2 \%$ in the same periods.

The SUPPORT trial [16] was a randomized, blinded, multicenter North American study enrolling infants born between 24 weeks and 0 days of gestation and 27 weeks and 6 days of gestation with an enrollment period from 2005 to 2009 . From the age of $2 \mathrm{~h}$ the low oxygen saturation target was $85-91 \%$ and the high target 91-95\%. Severe ROP was defined as threshold retinopathy, ophthalmologic surgery, or the use of bevacizumab treatment for retinopathy. The rate of infants with severe ROP was $17.9 \%$ in the high and $8.6 \%$ in the low saturation groups, respectively. Babies with a physiologic definition of BPD (receipt of more than $30 \%$ oxygen or the need for positive pressure support at 36 weeks) were $41.7 \%$ in the high and $38.0 \%$ in the low saturation arms. Death before discharge occurred in $107 / 662(16.2 \%)$ in the high and
$130 / 654(19.9 \%)$ in the low saturation groups [relative risk (RR) $1.27,95 \%$ CI 1.01-1.60; $\mathrm{p}=0.04$ ].

We therefore included a total of 8 articles with ROP as an outcome; of these only 1 is randomized and 7 are observational. The 8 included articles have BPD or lung complications as outcomes; of these, 3 report randomized studies and 5 report observational studies.

\section{Outcomes}

The primary outcome was severe ROP as defined in each study. Secondary outcomes were BPD/lung complications and survival.

\section{Statistical Methods}

We created a summary of the RR of ROP and BPD/lung complications comparing low versus high oxygen saturations. The average of the natural logarithm of the observed RR was estimated and the RR from each study was weighted by the inverse of its variance. We used random effects models, which take into account variation within and between studies (heterogeneity), to compute summary RRs [29]. A 2-tailed $\mathrm{p}<0.05$ was considered statistically significant. Heterogeneity between studies was evaluated with $\mathrm{Q}$ and $\mathrm{I}^{2}$ statistics [30]. For the Q statistic $\mathrm{p}<0.10$ was considered to indicate heterogeneity, as previously recommended [31]. $\mathrm{I}^{2}$ is the amount of total variation that is explained by variation between studies (i.e. $\mathrm{I}^{2}$ of 25,50 , and $75 \%$ indicate low, moderate, and high heterogeneity, respectively). Publication bias was assessed with Egger's test [32]. We also conducted sensitivity analyses excluding 1 study at a time to investigate whether the results were robust against the influence of single studies. Results from these sensitivity analyses are presented excluding the studies with the most positive and negative influences on the summary estimate.

\section{Results}

One randomized trial [16] and 7 observational studies $[12,20,24-28]$ including 3,811 patients investigated the association between $\mathrm{SpO}_{2}$ and the incidence of severe ROP (table 2). There was a significantly reduced incidence of severe ROP in the low $\mathrm{SpO}_{2}$ group with a summary RR of 0.42 (95\% CI 0.34-0.51) (figure 1). There was little evidence of heterogeneity $\left(\mathrm{I}^{2}=23 \%, \mathrm{p}=0.25\right)$.

There was a significant reduction both in the randomized trial (summary RR 0.48, 95\% CI 0.34-0.68) and among the 7 observational studies (summary RR 0.39, 95\% CI 0.30-0.51). When we further divided the observational studies into studies using historical controls and 'other studies' there was a similar association among studies using historical controls (summary RR 0.39, 95\% CI 0.26-0.58) and among the other studies (summary RR 0.36, 95\% CI 0.21-0.62) (results not shown). No single study explained the results, and the summary RR ranged from 0.39 (95\% CI 0.29-0.51) when excluding the study 


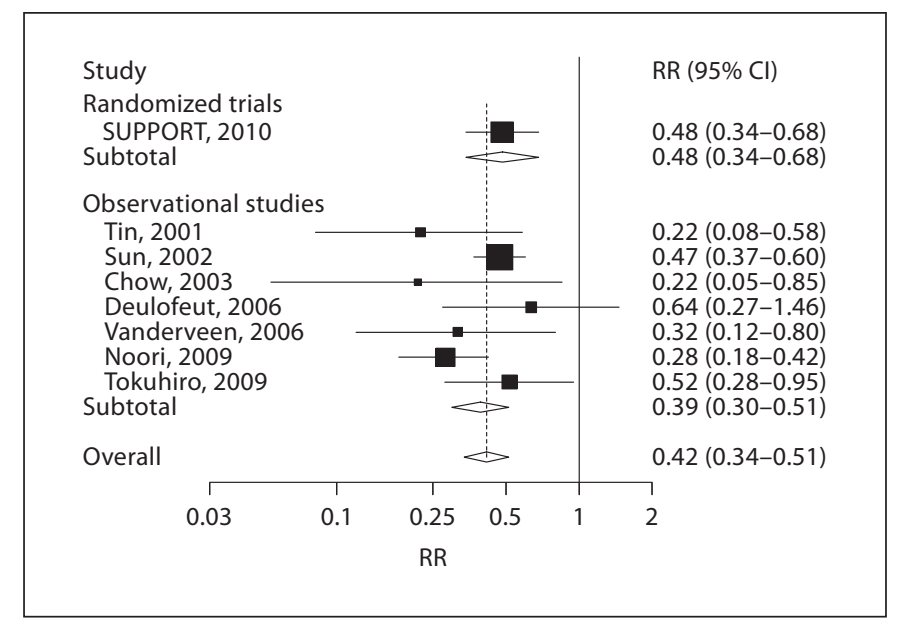

Fig. 1. RR and 95\% CI for randomized studies and observational studies as well as overall in 8 studies examining the effect on ROP of high versus low $\mathrm{SpO}_{2}$ in preterm infants. An $\mathrm{RR}<1$ favors low $\mathrm{SpO}_{2}$.

by Sun et al. [24] to 0.46 (95\% CI 0.39-0.55) when excluding the study by Noori et al. [20]. There was no evidence of publication bias ( $\mathrm{p}=0.29$ with Egger's test).

Three randomized trials $[16,22,23]$ and 5 observational studies [12, 20, 24, 25, 28] including 4,612 patients investigated the association between $\mathrm{SpO}_{2}$ and BPD (table 2). There was a significantly reduced incidence of BPD or lung problems in the low versus high saturation groups. The summary RR was 0.73 (95\% CI 0.63-0.86) (figure 2). There was high heterogeneity overall $\left(\mathrm{I}^{2}=68.0 \%, \mathrm{p}=\right.$ $0.003)$, but there was less heterogeneity among the 3 randomized trials $\left(\mathrm{I}^{2}=59.4 \%, \mathrm{p}=0.09\right)$; there was also a significant reduction in risk (summary RR $0.79,95 \%$ CI 0.64-0.97) for low versus high $\mathrm{SpO}_{2}$, while the summary RR was 0.70 (95\% CI 0.55-0.88) for the observational studies (figure 2). Further subdivision of the observational studies in studies with historical controls and 'other studies' showed summary RRs of 0.80 (95\% CI 0.57-1.13) and 0.56 (95\% CI 0.35-0.88), respectively, although these results are based on only $2-3$ studies in each subgroup. No single study explained the results and the summary RR ranged from 0.70 (95\% CI $0.60-0.82)$ when the study by Noori et al. [20] was excluded to 0.76 (95\% CI 0.66-0.89) when the study by Tin et al. [12] was excluded. There was no evidence of publication bias with Egger's test $(\mathrm{p}=0.48)$.

Death was registered in only 2 studies $[12,16]$ and the summary RR was 1.12 (95\% CI 0.86-1.45) (fig. 3) for low versus high $\mathrm{SaO}_{2}$. There was moderate, but not statistically significant, heterogeneity $\left(\mathrm{I}^{2}=56.9 \%, \mathrm{p}=0.13\right)$.

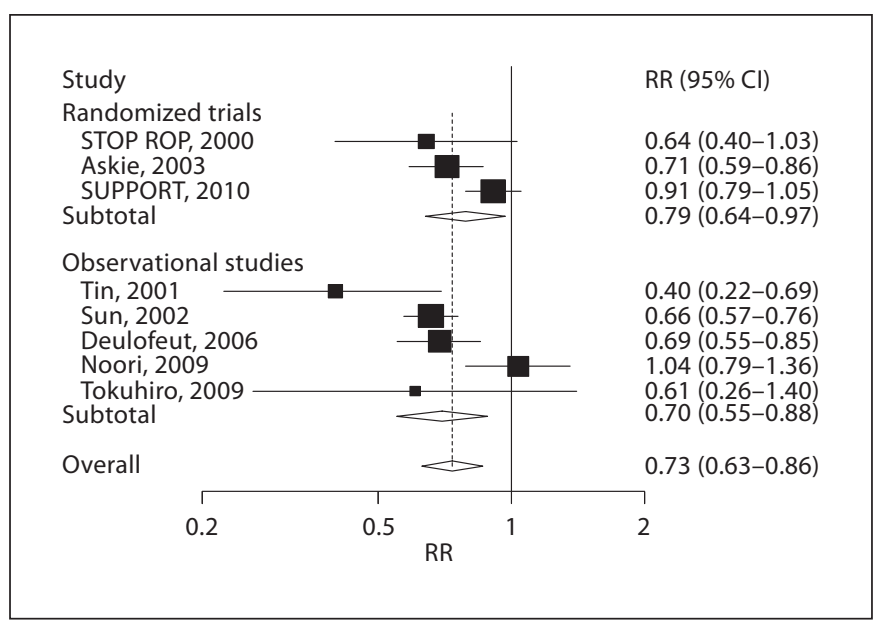

Fig. 2. RR and 95\% CI for randomized studies and observational studies as well as overall in 8 studies examining the effect on BPD and/or lung problems of high versus low $\mathrm{SpO}_{2}$ in preterm infants. An $\mathrm{RR}<1$ favors low $\mathrm{SpO}_{2}$.

Table 2. ROP and BPD/lung problems

\begin{tabular}{lccccc}
\hline $\begin{array}{l}\text { Study } \\
\text { (first author, year) }\end{array}$ & \multicolumn{2}{l}{ ROP } & & & \multicolumn{2}{c}{ BPD/lung problems } \\
\cline { 2 - 3 } \cline { 6 - 7 } & high & low & & high & low \\
\hline STOP-ROP, 2000 [22] & - & - & & $38 / 288$ & $25 / 295$ \\
Tin, 2001 [12] & $18 / 65$ & $4 / 65$ & & $30 / 65$ & $12 / 65$ \\
Sun, 2002 [24] & $161 / 742$ & $82 / 802$ & & $308 / 742$ & $219 / 802$ \\
Chow, 2003 [27] & $9 / 75$ & $2 / 77$ & & - & - \\
Askie, 2003 [23] & - & - & & $116 / 180$ & $82 / 178$ \\
Deulofeut, 2006 [25] & $16 / 221$ & $7 / 152$ & & $153 / 300$ & $71 / 202$ \\
Vanderveen, 2006 [26] & $44 / 251$ & $4 / 72$ & & - & - \\
Noori, 2009 [20] & $61 / 82$ & $18 / 86$ & & $61 / 136$ & $53 / 114$ \\
Tokuhiro, 2009 [28] & $19 / 59$ & $14 / 78$ & & $10 / 59$ & $8 / 78$ \\
SUPPORT, 2010 [16] & $91 / 509$ & $41 / 475$ & & $237 / 568$ & $205 / 540$ \\
\hline Total & $419 / 2,004$ & $172 / 1,807$ & & $953 / 2,338$ & $675 / 2,274$ \\
\hline
\end{tabular}

Number of ROP or BPD cases per total included.

\section{Discussion}

The data presented clearly show that both severe ROP and $\mathrm{BPD} /$ lung problems are reduced in premature infants with low instead of high oxygen saturation targeting.

There is a more than $50 \%$ reduction in severe ROP in low saturation targeted babies compared with high saturation targeted babies (9.5 vs. $20.9 \%)$. This is confirmed by the SUPPORT trial [16], the only randomized study 


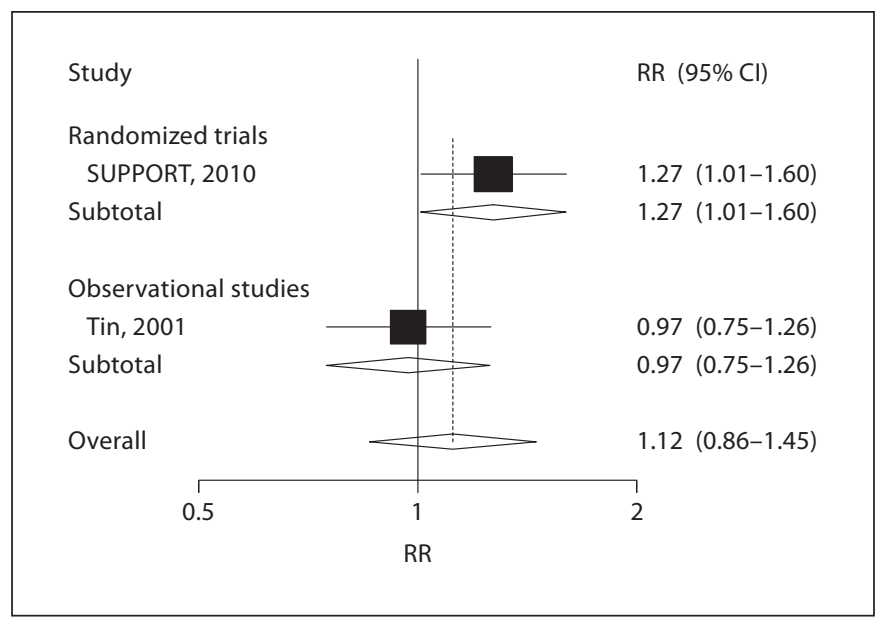

Fig. 3. RR and 95\% CI for 2 studies and overall examining the effect on survival of high versus low $\mathrm{SpO}_{2}$ in preterm infants. An $\mathrm{RR}<1$ favors low $\mathrm{SpO}_{2}$.

with this outcome. Our results are also in accordance with those of Chen et al. [15] who, based on 5 studies only, found in favor of low oxygen saturation an RR of 0.48 (95\% CI 0.31-0.75) for severe ROP compared with 0.42 (95\% CI 0.34-0.51) in the present study.

Further, we found that the incidence of BPD/lung problems was significantly reduced by $25 \%$ in the low saturation group in all studies ( 29.7 vs. $40.8 \%$ ) and by about $20 \%$ in the 3 randomized trials (30.8 vs. $37.7 \%$ ).

Our meta-analysis has several limitations which need to be discussed. Because there were few randomized controlled trials available we also included observational studies in this analysis. Although the observational studies reported on using different $\mathrm{SpO}_{2}$ ranges, they provided no data to prove that the infants has actually been treated within the given limits. Therefore, not only are many of the observational studies' results retrospective, but they also lack the certainty that no factors other than oxygen saturation differed between the groups. The use of surfactant, antenatal steroids, CPAP, and mechanical ventilation, to mention some factors, has substantially changed over time [33]. The use of historical controls therefore makes it difficult to predict whether the changes in outcomes are related to practice changes other than oxygen saturation targets between the different time periods. However, in addition to calculating summary estimates for all of the studies combined we also conducted analyses separately by study design (randomized vs. observational studies) but found significant inverse associa- tions among both study types both for ROP and BPD. Nevertheless, further randomized trials are needed to confirm or refute these results before any firm conclusions can be drawn.

There are also methodological problems linked to randomized trials. For instance, maintaining oxygen saturations within the preestablished targets seems difficult [34]. In the SUPPORT trial the median level of oxygen saturation in infants who received oxygen supplementation in the 2 treatment groups differed substantially. There was a considerable overlap between the groups and median levels were slightly higher than targeted levels in both treatment groups [16].

The populations investigated as well as the definition of high and low oxygen saturation differ between the studies. In this meta-analysis high and low $\mathrm{SpO}_{2}$ were used as defined in each article. However, it can be seen that the high groups in some of the latest studies are more or less identical to the low groups in the earliest studies. This illustrates a relatively dramatic change in attitude regarding $\mathrm{SpO}_{2}$ targeting in the most premature infants in the last decade with a significant reduction in saturation targets.

The definitions of BPD and lung problems also differ between the studies, yet all of the studies show that if babies are treated with a high saturation regime a higher rate of $\mathrm{BPD} /$ lung problems is found. A reduction of severe $\mathrm{ROP}$ of $50 \%$ and of $\mathrm{BPD} /$ lung problems of $20-25 \%$ in the smallest babies, as found in this review, indicates that tens of thousands of premature infants around the world escape BPD and severe ROP by the lowering of $\mathrm{SpO}_{2}$.

Already in the 1950s an association between increased mortality and restricting oxygen to prevent retrolental fibroplasia [35] was observed. In a recent Cocrhane review with a meta-analysis of 5 trials, the restriction of oxygen significantly reduced the incidence and severity of ROP without significantly increasing death rates [36].

In the present meta-analysis no difference in mortality was found; however, this result was based on only 2 studies, 1 of which was an observational study [12]. The SUPPORT trial found a 3.7\% absolute increased mortality in babies in the low $\mathrm{SpO}_{2}$ target group. This is a cause for concern and may indicate the need for a trade-off regarding $\mathrm{SaO}_{2}$ in order to reduce severe ROP without increasing mortality. However, mortality was not a primary outcome in that study. Further, the SUPPORT trial patients eligible but not randomized into the trial constituted a higher risk group than those who were enrolled. If these patients had been enrolled the final results, including mortality, could have been different [16]. We also 
know that the study enrolled only abut $25 \%$ of all eligible patients, which could have influenced the result. Thus any final conclusions regarding mortality cannot be drawn before the results from the other ongoing large randomized studies examining the association between $\mathrm{SpO}_{2}$ and mortality and morbidities are published. Perhaps a lower target of $85 \%$ is too low. Nevertheless, it should not be questioned any longer that a too high $\mathrm{SpO}_{2}$ of at least $>95 \%$ is detrimental.

Whether resuscitation/stabilization with a low $\mathrm{FiO}_{2}$ in the delivery room also reduces BPD is not yet clear but may also be a means to reduce BPD [37].

In spite of all of the research and publications in the last 10 years we still do not know the optimal $\mathrm{SpO}_{2}$ for the smallest and most immature babies. Moreover, we definitely do not know whether $\mathrm{SpO}_{2}$ should be increased at around 32 weeks of postconceptional age when children are prone to enter phase 2 of ROP. Both the STOP ROP trial [22] and the Boost trial [23] indicate that this will increase lung complications. Perhaps arterial oxygen saturation in the future should be monitored not only regarding markers of tissue hypoxia but also in relation to changes in growth factors such as insulin-like growth factor, vascular endothelial growth factor, and others which indicate phase 2 ROP development [38]. Until more data are accumulated the present guidelines should be followed [39].

In conclusion, we found a lower risk of ROP and BPD with low compared to high $\mathrm{SpO}_{2}$, and there was no difference in mortality. However, firm conclusions cannot be drawn before the results of several ongoing randomized trials testing short- and long-term outcomes of high versus low $\mathrm{SpO}_{2}$ targets are published.

\section{References}

1 Silverman WA: Retinopathy of prematurity: oxygen dogma challenged. Arch Dis Child 1982;57:731-733.

2 Hameed B, Shyamanur K, Kotecha S, Manktelow BN, Woodruff G, Draper ES, Field D: Trends in the incidence of severe retinopathy of prematurity in a geographically defined population over a 10-year period. Pediatrics 2004;113:1653-1657.

3 Manktelow BN, Draper ES, Annamalai S, Field D: Factors affecting the incidence of chronic lung disease of prematurity in 1987, 1992, and 1997. Arch Dis Child Fetal Neonatal Ed 2001;85:F33-F35.

4 Saugstad OD: Oxidative stress in the newborn - a 30-year perspective. Biol Neonate 2005; $88: 228-236$.

5 Bragt PC, Bonta IL: Oxidant stress during inflammation: anti-inflammatory effects of antioxidants. Agents Actions 1980;10:536539.

-6 McCord JM: A superoxide-activated chemotactic factor and its role in the inflammatory process. Agents Actions 1980;10:522-527.

7 Saugstad OD: Hypoxanthine as an indicator of hypoxia: its role in health and disease through free radical production. Pediatr Res 1988;23:143-150.

8 Saugstad OD: Oxygen and oxidative stress in bronchopulmonary Dysplasia. J Perinat Med 2010;38:571-577.

-9 Wardle SP, Drury J, Garr R, Weindling AM: Effect of blood transfusion on lipid peroxidation in preterm infants. Arch Dis Child Fetal Neonatal Ed 2002;86:F46-F48.

Optimal Oxygen Saturation for Extremely Low Birth Weight Infants
10 Lavoie PM, Claude Lavoie J, Watson C, Rouleau T, Chang BA, Chessex P: Inflammatory response in preterm infants is induced early in life by oxygen and modulated by TPN. Pediatr Res 2010;68:248-251.

11 Finer N, Leone T: Oxygen saturation monitoring for the preterm infant: the evidence basis for current practice. Pediatr Res 2009; 65:375-380.

12 Tin W, Milligan DW, Pennefather P, Hey E: Pulse oximetry, severe retinopathy, and outcome at one year in babies of less than 28 weeks gestation. Arch Dis Child Fetal Neonatal Ed 2001;84:F106-F110.

13 Saugstad OD: Optimal oxygenation at birth and in the neonatal period. Neonatology 2007;91:319-322.

14 Thomas W, Speer CP. Nonventilatory strategies for prevention and treatment of bronchopulmonary dysplasia - what is the evidence? Neonatology 2008;94:150-159.

15 Chen ML, Guo L, Smith LE, Dammann CE, Dammann O: High or low oxygen saturation and severe retinopathy of prematurity: a meta-analysis. Pediatrics 2010;125:e1483e1492.

16 SUPPORT Study Group of the Eunice Kennedy Shriver NICHD Neonatal Research Network, Carlo WA, Finer NN, Walsh MC, Rich W, Gantz MG, Laptook AR, et al: Target ranges of oxygen saturation in extremely preterm infants. N Engl J Med 2010;362: 1959-1969.

17 Wallace DK, Veness-Meehan KA, Miller WC: Incidence of severe retinopathy of prematurity before and after a modest reduction in target oxygen saturation levels. J AAPOS 2007;11:170-174.
18 Anderson CG, Benitz WE, Madan A: Retinopathy of prematurity and pulse oximetry: a national survey of recent practices. J Perinatol 2004;24:164-168.

19 Wright KW, Sami D, Thompson L, Ramanathan R, Joseph R, Farzavandi S: A physiologic reduced oxygen protocol decreases the incidence of threshold retinopathy of prematurity. Trans Am Ophthalmol Soc 2006; 104: 78-84.

20 Noori S, Patel D, Friedlich P, Siassi B, Seri I, Ramanathan R: Effects of low oxygen saturation limits on the ductus arteriosus in extremely low birth weight infants. J Perinatol 2009;29:553-557.

21 Sears JE, Pietz J, Sonnie C, Dolcini D, Hoppe $\mathrm{G}$ : A change in oxygen supplementation can decrease the incidence of retinopathy of prematurity. Ophthalmology 2009;116:513518.

22 Supplemental therapeutic oxygen for prethreshold retinopathy of prematurity (STOP-ROP), a randomized, controlled trial. 1. Primary outcomes. Pediatrics 2000;105: 295-310.

-23 Askie LM, Henderson-Smart DJ, Irwig L, Simpson JM: Oxygen-saturation targets and outcomes in extremely preterm infants. $\mathrm{N}$ Engl J Med 2003;349:959-967.

24 Sun SC: Relation of target $\mathrm{SpO}_{2}$ levels and clinical outcome in ELBW infants on supplementary oxygen (abstract). Pediatr Res 2002; 51:350A.

25 Deulofeut R, Critz A, Adams-Chapman I, Sola A: Avoiding hyperoxia in infants $<$ or $=$ $1,250 \mathrm{~g}$ is associated with improved shortand long-term outcomes. J Perinatol 2006; 26:700-705. 
-26 Vanderveen DK, Mansfield TA, Eichenwald EC: Lower oxygen saturation alarm limits decrease the severity of retinopathy of prematurity. J AAPOS 2006;10:445-448.

27 Chow LC, Wright KW, Sola A, CSMC Oxygen Administration Study Group: Can changes in clinical practice decrease the incidence of severe retinopathy of prematurity in very low birth weight infants? Pediatrics 2003;111:339-345.

-28 Tokuhiro Y, Yoshida T, Nakabayashi Y, Nakauchi S, Nakagawa Y, Kihara M, Mitsufuji N, Kizaki Z: Reduced oxygen protocol decreases the incidence of threshold retinopathy of prematurity in infants of $<33$ weeks gestation. Pediatr Int 2009;51:804-806.

-29 DerSimonian R, Laird N: Meta-analysis in clinical trials. Control Clin Trials 1986;7: 177-188.
30 Higgins JP, Thompson SG: Quantifying heterogeneity in a meta-analysis. Stat Med 2002; 21:1539-1558.

31 Dickersin K, Berlin JA: Meta-analysis: stateof-the-science. Epidemiol Rev 14:1992;154176.

32 Egger M, Davey SG, Schneider M, Minder C: Bias in meta-analysis detected by a simple, graphical test. BMJ 1997:315:629-634.

33 Morley CJ: CPAP and low oxygen saturation for very preterm babies? N Engl J Med 2010; 362:2024-2026.

34 Rich WD, Auten KJ, Gantz MG, Hale EC, Hensman AM, Newman NS, Finer NN, National Institute of Child Health and Human Development Neonatal Research Network: Antenatal consent in the SUPPORT trial: challenges, costs, and representative enrollment. Pediatrics 2010;126:e215-e221.

35 Bolton DP, Cross KW: Further observations on cost of preventing retrolental fibroplasia. Lancet 1974;1:445-448.
36 Askie LM, Henderson-Smart DJ, Ko H: Restricted versus liberal oxygen exposure for preventing morbidity and mortality in preterm or low birth weight infants. Cochrane Database Syst Rev 2009;21:CD001077.

37 Vento M, Moro M, Escrig R, Arruza L, Villar G, Izquierdo I, et al: Preterm resuscitation with low oxygen causes less oxidative stress, inflammation, and chronic lung disease. Pediatrics 2009; 124:e439-449.

38 Chen J, Smith LE: Retinopathy of prematurity. Angiogenesis 2007;10:133-140.

39 Sweet DG, Carnielli V, Greisen G, Hallman M, Ozek E, Plavka R, Saugstad OD, Simeoni U, Speer CP, Halliday HL, European Association of Perinatal Medicine: European consensus guidelines on the management of neonatal respiratory distress syndrome in preterm infants - 2010 update. Neonatology 2010;97:402-417. 